\title{
A case study: A leader's commitment to transparency and accountability through a serious patient harm event
}

\author{
J eanette I ves Erickson, Marianne Ditomassi, Theresa Gallivan, Keith Perleberg, Mary Jane Costa \\ Nursing and Patient Care Services, Massachusetts General Hospital, Boston, MA, USA
}

Correspondence: Jeanette Ives Erickson. Address: Massachusetts General Hospital, 55 Fruit Street, BUL-230B, Boston, MA 02114, USA. E-mail: jiveserickson@partners.org

Received: October 19, 2012

Accepted: January 27, 2013

Online Published: February 5, 2013

DOI : 10.5430/jha.v2n3p1

URL: http://dx.doi.org/10.5430/jha.v2n3p1

\begin{abstract}
Analysis reveals that most preventable adverse events result from systemic causes, not human error. The senior patient care executive at a leading hospital recounts the unnecessary death of a patient and the investigation and personal learning that followed. This case study offers a blueprint for leaders managing a serious patient harm event.
\end{abstract}

\section{Key words}

Patient harm, Patient death, Just culture

\section{I ntroduction}

The majority of factors giving rise to serious reportable events are systemic; that is, they are not the result of poorly performing individual nurses, physicians, or other providers ${ }^{[1]}$. As the chief nurse at Massachusetts General Hospital, (MGH), these words took on a deeply personal meaning in 2009 when a patient unnecessarily died on my watch.

On postoperative day number three, the patient was continuing his recovery on an inpatient medical surgical unit. A cardiac monitor continually observed his heart rate at the same time a pulse oximeter measured his oxygen saturation level. While making rounds, the nurse found the patient to be not breathing and he did not have a pulse. She immediately began administering cardiopulmonary resuscitation (CPR) and a code was called. Unfortunately, we were not able to resuscitate the patient.

Following the code, the nursing staff was searching for an explanation as to why they had not heard the heart monitor alarms. What we discovered shocked us and our entire organization. To our dismay, we found out that a lethal arrhythmia had gone undetected for several minutes and had killed the patient - the patient's monitor alarm had been turned off.

My first step was to ensure that everyone knew the incident signaled a human-system interface failure - not one of an individual person. The family was informed, and a communication and education plan was implemented. We spent the next six hours disabling each of the hospital's 1,100 monitors' "off" switches. My involvement could have ended here, but it did not. 


\section{Preparing media talking points}

It was not long after this unfortunate event that a reporter from the Boston Globe called for an interview. My immediate reaction was "No way!" Nonetheless, the senior vice president for Quality and Safety and I began collaborating to create a set of specific "talking points" which became essential to our ability to communicate both internally and externally. However, the media reporters continued to search for someone to blame. One reporter asked repeatedly, "Who did this?" I tried to convey to the reporter that our systems were at fault - not our nurses. When she insisted on a name, I gave her mine, requesting only that she spell it correctly, and print it in bold capital letters.

\section{Supporting a just culture}

To me, it was important that no one in this organization asked, "Who did this?" In my view, pointing to human error as a cause rather than a consequence serves as a cloak for ignorance. Ensuring that the error does not become the discussion's end point is central to the framework for an important reason. Ending the discussion at the point of blame prevents learning.

Each year in the United States, treatment errors injure an estimated 1,000,000 patients, as many as 98,000 of whom die from the injury. This injury rate, which is three times greater than the number of Americans who die in automobile accidents each year, carries a significant price tag as well - as much as USD $\$ 33$ billion ${ }^{[1]}$. One expert maintains that the single greatest impediment to error prevention is the fact that "we punish people for making mistakes" ${ }^{[2]}$. He strongly recommends that hospitals abandon their existing, punitive error reporting systems to make reporting "safe" for healthcare professionals and hospital staff. Creating a just culture - "an atmosphere of trust in which people are encouraged for providing essential safety-related information, but in which they are also clear about where the line must be drawn between acceptable and unacceptable behavior" - helps to create and sustain a safe reporting environment ${ }^{[3]}$. Principles of a just culture include:

a) Ensuring frontline personnel feel comfortable disclosing errors - including their own —while maintaining professional accountability.

b) Not holding individual practitioners accountable for system failings over which they have no control.

c) Recognizing many individual or "active" errors represent predictable interactions between human operators and the systems in which they work.

d) Revising health care's culture that used to hold individuals accountable for all errors or mishaps that befall patients under their care.

e) Not tolerating conscious disregard of clear risks to patients or gross misconduct (e.g., falsifying a record, performing professional duties while intoxicated) ${ }^{[3]}$.

Effective reporting systems strike a careful, considered balance between deterrence and communication. Answering two seminal questions will help steady that delicate balance:

i. If the threat of discipline increases one's awareness of risk or at least increases one's interest in assessing the risks, does this heightened awareness outweigh the learning that comes from thorough error investigation?

ii. If an employee makes a mistake, can that employee safely come forward so that the organization can learn from the event?

An integral part of that just culture requires that post-mishap investigations integrate human factors theory. 


\section{Integrating Human Factors Theory}

In looking at the circumstances around this patient harm event, human factors theory quickly became important. Human factor theory research centers on how human beings interact at work and in daily living with products, devices, procedures, work spaces, and the environments encountered.

An understanding of human factors theory comes into play when purchasing new technology, designing new roles and conducting safety rounds. The definition states, "Human factors research discovers and applies information about human behavior, abilities, limitations, and other characteristics to the design of tools, machines, systems, tasks, and jobs, and environments for productive, safe, comfortable, and effective human use" ${ }^{[4]}$. I enlisted an expert to teach this thinking to my entire leadership team. The following, see Figure 1, is a very useful graphic that is closely aligned with my belief system and how I have decided to approach the work ahead of me.

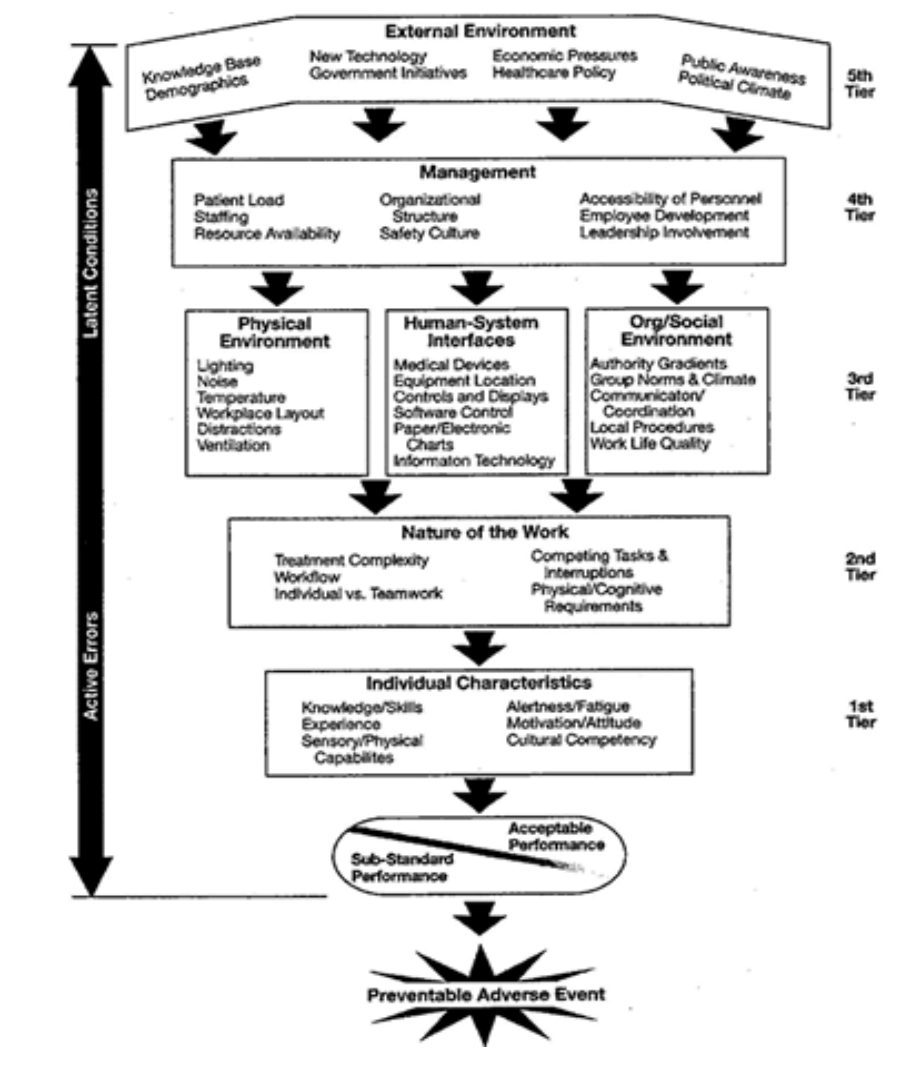

Figure 1. Contributing Factors to Adverse Events in Health Care ${ }^{[5]}$

In brief, many patient harm events result from this unique interaction or alignment of several necessary but singly insufficient factors. Weaknesses in these factors typically are present in the system long before the occurrence of an adverse event. All that is needed is for a sufficient number to become aligned for a serious patient harm event to occur ${ }^{[5]}$.

This illustration from and comment by the Agency for Healthcare Research and Quality puts into perspective the patient harm event that happened on my watch ${ }^{[5]}$. Critically reviewing each tier of the diagram guided the MGH leadership and clinical teams in identifying the human-system interface failure which factored into this patient harm situation. We were then able to focus our attention on the development of workgroups to develop policies and educational programs to ensure that a like incident was prevented in the future. 


\section{Demonstrating transparency as a value}

I've come to see transparency not as a management approach, but as a value system. One cannot be selectively transparent; either decision making is public or it is not. When it came to discussing, investigating and resolving the root causes of this sentinel event, all draft documents, all arguments for and against my proposals, the decisions about the decision-making process itself, and all final decisions, have been made public.

As we moved through the analysis of this patient harm event, I communicated my findings at meetings of trustees, chiefs, leadership teams and open forums. I wanted everyone to learn about what happened and why. This open approach prompted the Chief Executive Officer to give me responsibility for defining the hospital's response plan. Not only has it allowed me to obtain timely feedback, I've been able to publicly demonstrate the pivotal role of nursing in the quality and safety arena to the hospital-at-large.

\section{Mapping care delivery}

In order to "map" what happened in this case study, I reviewed our patient care delivery model. The MGH Patient Care Delivery Model represents the convergence of our mission, vision, values and long-range goals to support the delivery of patient care. Our model depicts the dynamic and therapeutic interaction that occurs between the professional care provider and the patient - especially in relation to health and illness. An open, evolving and interactive system, the model centers on a continuous exchange between the clinician and the patient. The model also captures the internal and external forces that influence the patient's experience as well as the structures supporting the delivery of patient care.

Figure 2. MGH Patient Care Delivery Model (C) MGH Nursing and Patient Care Services, 1997

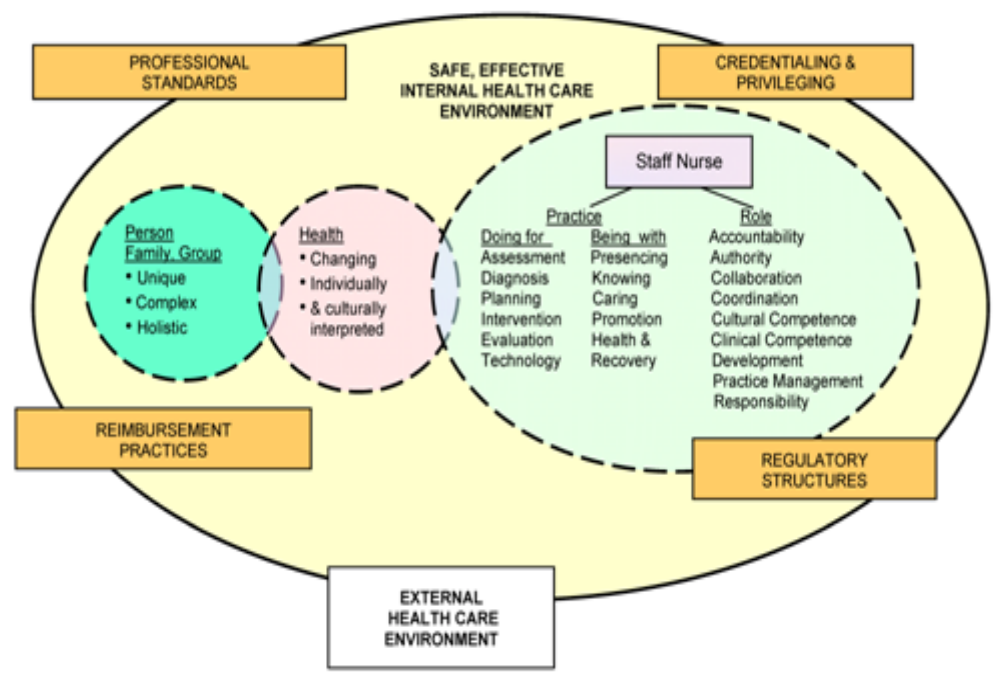

The model contains three major components. The inner circles depict the dynamic interaction between person, health and clinician. The lines of the internal circles are interrupted to represent the open and fluid nature of the model. The larger circle surrounding the core represents the interdisciplinary partnerships within the multiple settings where patient care is delivered. The internal forces reflect the vision, guiding principles and our long-range goals. The external forces are depicted as the components of professional standards, credentialing and privileging standards, regulatory structures, and reimbursement practices. These forces continuously interact with the internal environment and the inner core.

One might say that this is a nice conceptual model. The real problem with this is that it is not a model that was developed with our physician colleagues or with patients. We have numerous patient and family advisory committees whom I 
subsequently met with to tell them about the death of this patient and to share with them how we were going about reviewing our systems. I recognized that their input was essential to the development of a comprehensive plan.

To promote interdisciplinary collaboration to identify recommendations to improve physiologic monitoring systems and responses to ensure a safe, evidence-based, and reliable monitoring program for patients, a multi-faceted interdisciplinary workgroup structure (Figure 3) led by an Associate Chief Nurse and the Assistant Chief for Quality Assurance in Primary Care. Charges included:
A. Critically reviewing MGH practice standards, evidence-based practice and research on physiologic monitoring and alarm management;
B. Making recommendations regarding criteria for, and management of, physiologic monitoring; and,
C. Reviewing recommendations for patient placement standards/criteria.

The authors cite that key lessons learned during this important interdisciplinary work included "the importance of coordinating the various departments into an effective team, and, secondly, recognizing the utility of shifting emphasis from time to time of the relative importance of various departments to the business."

Figure 3. Physiologic Monitoring Team Workgroups and Deliverables (C) Massachusetts General Hospital, 2011

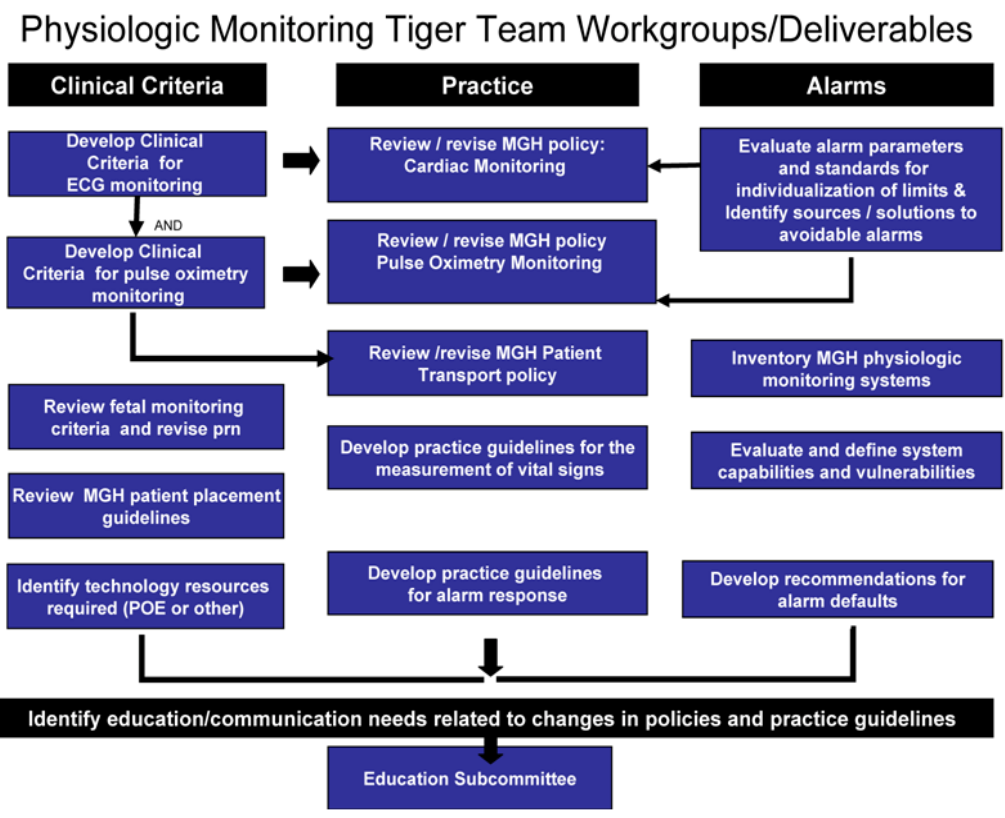

\section{Heightening awareness of human system interfaces}

While a scarcity of evidence-based research prevails currently, the American Heart Association's Practice Standards for Electrocardiographic Monitoring in Hospital Setting serves as a compendium of best practices related to alarm monitoring ${ }^{[6]}$. The recommendations for the maintenance and improvement of quality care in cardiac monitoring include the following:

- Establish protocols that govern the roles and responsibilities of all levels of staff regarding cardiac monitoring, documentation of ECG changes, periodic documentation that alarms are set appropriately, and response to emergency and non-emergency events. 
- Institute a mandatory comprehensive orientation and training program that includes all staff involved in cardiac monitoring with demonstrated competences in specific cardiac monitoring skills (first shock for life-threatening arrhythmias, diagnostic accuracy of rhythm interpretations, appropriateness and quality of 12-lead ECGs, timeliness of human review of computer-generated alarms, and rhythm strips).

- Conduct a mandatory periodic competency evaluation of all staff to ensure continued proficiency in critical elements of cardiac monitoring - periodic audits of electrode placement and rhythm strip interpretation.

- Provide continuing education to reinforce current knowledge, and update staff on research findings and techniques.

- Conduct a periodic review of unit protocols, initial training, staff competency level, and ongoing education efforts to determine whether they continue to meet staff and patient needs, to include reviews of staff performance, critical events, and patient outcomes.

While these recommendations represent a strong first step towards more effectively using the sophisticated alarm monitors, additional research is needed to identify opportunities to further improve performance in two critical areas: reducing the frequency of "nuisance" alarms and combating alarm fatigue.

\section{Conclusion}

Effective leaders learn to deal with and anticipate the unexpected ${ }^{[7]}$. As nurses and executives, we understand that threats - some anticipated and many unexpected - permeate healthcare delivery. I hope that through my reflections, new learning, plans and actions, a new environment of mindfulness will emerge. In that culture, we will design systems with the requirements and expectations of patients and their families in mind. In my view, our heightened state of awareness and transparency will improve our ability to acknowledge, correct and redesign flawed systems, which is critical to identifying errors before the next crisis occurs.

Not only has this experience helped me grow professionally and personally, it has transformed the way I communicate with my clinical staff, chiefs of service and executive colleagues. My experience confirmed what I already knew in my heart - the right responses to adverse events are always transparent, proactive, and patient-centered. In my quest for transparency, I wanted to offer the details of our experience to nurse leaders everywhere. This article is part of that effort to encourage others in the healthcare delivery system to leverage our experience for the benefit of themselves and their patients.

\section{References}

[1] Kohn LT, Corrigan JM, Donaldson MS, eds. To err is human: building a safer health system. A report of the Committee on Quality of Health Care in America, Institute of Medicine. Washington, D.C.: National Academy Press; 2000.

[2] Leape L, MD. Testimony, United States Congress, House Committee on Veterans' Affairs, 1997.

[3] Marx D. Patient Safety and the "Just Culture:" A Primer for Health Care Executives, Columbia University, 2001.

[4] Chapanis A, Garner W, Morgan C. Applied Experimental Psychology: Human Factors in Engineering Design. New York: Wiley; 1985.

[5] Henricksen K, Dayton E, Keyes M, Carayon P. Understanding Adverse Events: A Human Factors Framework. In: Hughes R, ed. Patient Safety and Quality: An Evidence-Based Handbook for Nurse. 2008; 1-13. Rockville, MD: Agency for Healthcare Research and Quality (US). http://dx.doi.org/10.1161/01.CIR.0000145144.56673.59

[6] Drew BJ, Califf RM, Funk M, Kaufman ES, Krucoff MW, Laks PW, Hare GF. Practice standards for electrocardiographic monitoring in hospital settings: an American Heart Association scientific statement from the Councils on Cardiovascular Nursing, 
Clinical Cardiology, and Cardiovascular Disease in the Young. Circulation. 2004; 110: 2721-2746. PMid:15505110 http://dx.doi.org/10.1161/01.CIR.0000145144.56673.59

[7] Weick KE, Sutcliffe KN. Managing the Unexpected: Assuring High Performance in an Age of Complexity. New York: John Wiley \& Sons, 2001. PMid:11494412 